\title{
Product Hardy Operators on Hardy Spaces
}

\author{
Dashan FAN and Fayou ZHAO \\ University of Wisconsin-Milwaukee and Shanghai University \\ (Communicated by Y. Furuya)
}

\begin{abstract}
We study the product Hausdorff operator $H_{\Phi}$ on the product Hardy spaces, and prove that, for a nonnegative valued function $\Phi, H_{\Phi}$ is bounded on the product Hardy space $H^{1}(\mathbb{R} \times \mathbb{R})$ if and only if $\Phi$ is a Lebesgue integrable function on $(0, \infty) \times(0, \infty)$. As an application, we know that the product Hardy operator $\mathcal{H}$ is not bounded on $H^{1}(\mathbb{R} \times \mathbb{R})$. On the other hand, we prove that $\|\mathcal{H} f\|_{H^{1}(\mathbb{R} \times \mathbb{R})} \preceq\|f\|_{H^{1}(\mathbb{R} \times \mathbb{R})}$ if $f$ is an even function. Furthermore, using the $H^{p}(\mathbb{R} \times \mathbb{R})$ boundedness criterion of Fefferman, we prove that the $k$-th order Hardy operator is bounded on $H^{p}(\mathbb{R} \times \mathbb{R})$ whenever $k>1 / p-1$.
\end{abstract}

\section{Introduction}

Let $\Phi$ be a suitable function. The classical one-parameter Hausdorff operator with the kernel function $\Phi$ is defined in the dilation-integral form by

$$
h_{\Phi}(f)(x)=\int_{0}^{\infty} \frac{\Phi(t)}{t} f\left(\frac{x}{t}\right) d t,
$$

where, for the sake of simplicity, we initially assume that the operator acts on the class of Schwartz functions $f$. The Hausdorff operator $h_{\Phi}$ is an important operator on analysis and its boundedness on various function or distribution spaces was extensively studied in recent years. The reader can see [1,2, 9-16] to find a lot of results and historical developments about this operator. As an analog, the product Hausdorff operator

$$
H_{\Phi}(f)(x, y)=\int_{0}^{\infty} \int_{0}^{\infty} \frac{\Phi(s, t)}{s t} f\left(\frac{x}{s}, \frac{y}{t}\right) d s d t
$$

is also received some attention (see [19, 20]). Particularly, if one chooses

$$
\Phi(s, t)=\frac{\chi_{(1, \infty) \times(1, \infty)}(s, t)}{s t},
$$

Received December 15, 2013; revised February 4, 2014 2010 Mathematics Subject Classification: 42B20, 42B25, 42B35

Key words and phrases: Product Hausdorff operators, Product Hardy operator, Product Hardy spaces, Atomic decomposition, Hilbert transforms

The research was supported by the NNSF of China (Grant Nos. 11201287, 11471288). 
then the operator $H_{\Phi}$ becomes the product Hardy operator

$$
\mathcal{H} f(x, y)=\frac{1}{x y} \int_{0}^{x} \int_{0}^{y} f(t, s) d t d s
$$

that is also an interesting operator in harmonic analysis [8, 17, 18, 20].

When $p>1$, by the Minkowski integral inequality and changing variables on the $L^{p}(\mathbb{R} \times \mathbb{R})$ integral, we obtain

$$
\begin{aligned}
\left\|H_{\Phi}(f)\right\|_{L^{p}(\mathbb{R} \times \mathbb{R})} & \leq \int_{0}^{\infty} \int_{0}^{\infty} \frac{|\Phi(s, t)|}{s t}\left\|f\left(\frac{\dot{-}}{s}, \frac{\dot{t}}{t}\right)\right\|_{L^{p}(\mathbb{R} \times \mathbb{R})} d s d t \\
& =\|f\|_{L^{p}(\mathbb{R} \times \mathbb{R})} \int_{0}^{\infty} \int_{0}^{\infty} \frac{|\Phi(s, t)|}{s t} s^{1 / p} t^{1 / p} d s d t
\end{aligned}
$$

It indicates that $H_{\Phi}(f)$ is bounded on the Lebesgue space $L^{p}(\mathbb{R} \times \mathbb{R})$, if

$$
\int_{0}^{\infty} \int_{0}^{\infty} \frac{|\Phi(s, t)|}{s t} s^{1 / p} t^{1 / p} d s d t<\infty .
$$

When $0<p \leq 1$, a good substitution of $L^{p}$ is the Hardy space $H^{p}$. The space $H^{1}$ is a subspace of $L^{1}$ and it is still a norm space. Thus, the Minkowski integral inequality gives

$$
\left\|H_{\Phi}(f)\right\|_{H^{1}(\mathbb{R} \times \mathbb{R})} \leq \int_{0}^{\infty} \int_{0}^{\infty} \frac{|\Phi(s, t)|}{s t}\left\|f\left(\frac{\cdot}{s}, \frac{\cdot}{t}\right)\right\|_{H^{1}(\mathbb{R} \times \mathbb{R})} d s d t .
$$

Now an easy scaling argument shows that

$$
\left\|f\left(\frac{\dot{-}}{\bar{s}}, \overline{\bar{t}}\right)\right\|_{H^{1}(\mathbb{R} \times \mathbb{R})}=s t\|f\|_{H^{1}(\mathbb{R} \times \mathbb{R})} .
$$

Hence, we know that $H_{\Phi}$ is bounded on $H^{1}(\mathbb{R} \times \mathbb{R})$, provided

$$
\int_{0}^{\infty} \int_{0}^{\infty}|\Phi(s, t)| d s d t<\infty .
$$

Furthermore, in the following theorem, we will show that the above condition on $\Phi$ is also a necessity condition, if $\Phi$ is nonnegative valued.

Theorem 1. Assume that $\Phi$ is a nonnegative valued function. Then $H_{\Phi}$ is bounded on $H^{1}(\mathbb{R} \times \mathbb{R})$ if and only if $\Phi(s, t) \in L^{1}\left(\mathbb{R}_{+} \times \mathbb{R}_{+}\right)$.

Note that the kernel function $\Phi(s, t)=\frac{\chi_{(1, \infty) \times(1, \infty)}(s, t)}{s t}$ of the product Hardy operator is not Lebesgue integrable. Thus by Theorem 1, we know that the product Hardy operator $\mathcal{H}$ is not bounded on $H^{1}(\mathbb{R} \times \mathbb{R})$. But if $f \in H^{1}(\mathbb{R} \times \mathbb{R})$ is even, then we have the following result.

Theorem 2. If $f(x, y) \in H^{1}(\mathbb{R} \times \mathbb{R})$ is even on both $x$ and $y$ variables, then we have

$$
\|\mathcal{H} f\|_{H^{1}(\mathbb{R} \times \mathbb{R})} \preceq\|f\|_{H^{1}(\mathbb{R} \times \mathbb{R})} .
$$


Additionally, recent papers $[2,14,20]$ addressed the boundedness of $H_{\Phi}$ on the Hardy space $H^{1}(\mathbb{R} \times \mathbb{R})$, Besov space $B_{0}^{1,0}(\mathbb{R} \times \mathbb{R})$ and the local Hardy space $h^{1}(\mathbb{R} \times \mathbb{R})$, as well as the $H^{1}(\mathbb{R} \times \mathbb{R}) \rightarrow L^{1}(\mathbb{R} \times \mathbb{R})$ boundedness of $H_{\Phi}$. However, we are not able to find any paper in the literature to study the boundedness of $H_{\Phi}$ on the product Hardy spaces $H^{p}(\mathbb{R} \times \mathbb{R})$ for $0<p<1$. This problem seems interesting and involved. First, $H^{p}(\mathbb{R} \times \mathbb{R})$ is merely a quasi-norm space. The argument involving the Minkowski integral inequality to treat the $H^{1}$ case does not work in this case. More significantly, the dual space and atomic structure of the product Hardy space $H^{p}(\mathbb{R} \times \mathbb{R})$ is much more complicated, compared to those of the one parameter Hardy space $H^{p}(\mathbb{R})$. Thus, all methods used to study the $H^{p}(\mathbb{R})$ boundedness of $h_{\Phi}($ see $[9,11,15])$ fail in studying $H^{p}(\mathbb{R} \times \mathbb{R})$ boundedness of $H_{\Phi}$. Motivated by above observations, in order to study the $H^{p}(\mathbb{R} \times \mathbb{R})$ boundedness of $H_{\Phi}$, in this paper we will work on a special case of $H_{\Phi}$, the $k$-th product Hardy operator

$$
\mathcal{H}_{(k)} f(x, y)=\frac{1}{x^{k+1} y^{k+1}} \int_{0}^{x} \int_{0}^{y} t^{k} s^{k} f(t, s) d t d s .
$$

By a changing of variables, we can write

$$
\mathcal{H}_{(k)} f(x, y)=\int_{1}^{\infty} \int_{1}^{\infty} \frac{1}{t^{k+2} s^{k+2}} f(x / t, y / s) d t d s .
$$

Hence, the $k$-th order product Hardy operator is also a special case of the product Hausdorff operator if we set

$$
\Phi(s, t)=\frac{\chi(1, \infty) \times(1, \infty)(s, t)}{s^{k+1} t^{k+1}} .
$$

So, if $k=0$ then $\mathcal{H}_{(0)}$ is just the product Hardy operator. We will establish the following result.

Theorem 3. $\mathcal{H}_{(k)}$ is bounded on $H^{p}(\mathbb{R} \times \mathbb{R})$ if $k>1 / p-1$.

We will adopt some basic ideas used in one parameter case [5] to prove Theorem 1 and Theorem 2. However, the argument here is more technically difficult to execute in order to treat the product case. Furthermore, to prove Theorem 3, we will invoke a famous $H^{p}(\mathbb{R} \times$ $\mathbb{R}$ ) boundedness criterion established by R. Fefferman. This criterion and some preliminary knowledge will be recalled in Section 2. Finally we prove these theorems in Section 3.

Throughout this paper, we use the notation $A \simeq B$ if there exist positive constants $C$ and $c$, independent of all essential variables such that

$$
c B \leq A \leq C B .
$$

Also, we use the notation $A \preceq B$ to mean that there is a constant $C>0$ independent of all essential variables such that $A \leq C B$. 


\section{Some Preliminary Knowledge}

We start with briefly describing the definition of the product Hardy spaces. Let $\phi \in S(\mathbb{R})$ and $\varphi \in S(\mathbb{R})$ satisfy

$$
\int_{\mathbb{R}} \phi(x) d x \int_{\mathbb{R}} \varphi(y) d y \neq 0
$$

and denote

$$
\phi_{t}(x)=\frac{1}{t} \phi\left(\frac{x}{t}\right), \varphi_{s}(y)=\frac{1}{s} \varphi\left(\frac{y}{s}\right), \quad s, t>0 .
$$

The product Hardy space $H^{p}(\mathbb{R} \times \mathbb{R})$ is the space of all distributions $f$ satisfying

$$
\|f\|_{H^{p}(\mathbb{R} \times \mathbb{R})}:=\left\|\sup _{0<s, t<\infty}\left|\left(\phi_{t} \otimes \varphi_{s}\right) * f\right|\right\|_{L^{p}(\mathbb{R} \times \mathbb{R})}<\infty,
$$

where

$$
\left(\phi_{t} \otimes \varphi_{s}\right)(x, y)=\phi_{t}(x) \varphi_{s}(y) .
$$

It is known that the definition of $H^{p}$ is flexible on the choice of the functions $\phi$ and $\varphi$.

Compared to the one parameter atomic Hardy space (see [3]), the atomic decomposition of the product Hardy space is quite complicated. The following content can be found in [4].

Let $R$ denote a rectangle and $\gamma R$ the rectangle with the same center of $R$ and its side length is $\gamma$-times of the side length of $R$. For an open set $\Omega \subset \mathbb{R}^{2}$ of finite measure, let $m(\Omega)$ denote the set of all maximal dyadic rectangles in $\Omega$. Assume $0<p \leq 1$, and $s$ is any integer larger than or equal to $[1 / p-1]$, the integer part of $1 / p-1$. A function $A(x, y)$ is called a $(p, s)$-atom if $A$ satisfies the following three conditions:

(1) $\operatorname{supp}(A) \subset \Omega$, where $\Omega \subset \mathbb{R}^{2}$ is an open set of finite measure;

(2) A can be written as

$$
A=\sum_{R \in m(\Omega)} a_{R}
$$

where each $a_{R}$ satisfies

(2-i) $\operatorname{supp}\left(a_{R}\right) \subset 3 R$,

$$
\int_{\mathbb{R}} x^{\alpha} a_{R}(x, y) d x=0, \quad \text { all } y \in \mathbb{R}
$$

and 


$$
\int_{\mathbb{R}} a_{R}(x, y) y^{\alpha} d y=0, \text { all } x \in \mathbb{R}
$$

for $\alpha=0,1,2, \ldots, s$;

(3)

$$
\|A\|_{L^{2}(\mathbb{R} \times \mathbb{R})} \leq|\Omega|^{1 / 2-1 / p}, \quad \sum_{R \in m(\Omega)}\left\|a_{R}\right\|_{L^{2}(\mathbb{R} \times \mathbb{R})}^{2} \leq|\Omega|^{1-2 / p} .
$$

It is known that for $f \in H^{p}(\mathbb{R} \times \mathbb{R}), f$ can be written as

$$
f=\sum \lambda_{j} A_{j}
$$

where each $A_{j}$ is a $(p, s)$-atom and

$$
\sum\left|\lambda_{j}\right|^{p} \simeq\|f\|_{H^{p}(\mathbb{R} \times \mathbb{R})}^{p} .
$$

Also, to study the Hausdorff operator on $H^{p}(\mathbb{R} \times \mathbb{R})$, we will employ the Fefferman's criterion for $H^{p}(\mathbb{R} \times \mathbb{R})$ boundedness of linear operators with the rectangular atoms. Here, a function $a(x)$ is called a rectangular $(p, 2, s)$-atom if it satisfies the following three conditions:

(a) $\operatorname{supp}(a) \subset R=I \times J$, where $I$ and $J$ are intervals in $\mathbb{R}$;

(b)

$$
\int_{\mathbb{R}} x^{\alpha} a(x, y) d x=0 \text { for all } y \in \mathbb{R}
$$

and

$$
\int_{\mathbb{R}} a(x, y) y^{\alpha} d y=0 \text { for all } x \in \mathbb{R}
$$

for $\alpha=0,1,2, \ldots, s$, where $s$ is a fixed integer larger than or equal to $[1 / p-1]$;

(c)

$$
\|a\|_{L^{2}(\mathbb{R} \times \mathbb{R})} \leq|R|^{1 / 2-1 / p} .
$$

For a rectangular $(p, 2, s)$-atom, using Hölder's inequality, we have

$$
\int_{\mathbb{R} \times \mathbb{R}}|a(x, y)|^{p} d x d y \preceq\|a\|_{L^{2}}^{p}|R|^{\frac{1-p}{2}} \leq 1 .
$$

The following theorem is well known.

Theorem A (R. Fefferman [6, 7]). Let $T$ be a linear operator bounded on $L^{2}(\mathbb{R} \times$ $\mathbb{R})$. Suppose there exist positive numbers $C$ and $\delta$, for any rectangular $(p, 2, s)$-atom a with 
support in $R$,

$$
\int_{(\gamma R)^{C}}|T(a)(x, y)|^{p} d x d y \leq C \gamma^{-\delta}
$$

holds for all $\gamma \geq 2$. Then the operator T is bounded from $H^{p}(\mathbb{R} \times \mathbb{R})$ to $L^{p}(\mathbb{R} \times \mathbb{R})$.

The space $H^{p}(\mathbb{R} \times \mathbb{R})$ also can be characterized by using the Hilbert transforms. We define three Hilbert transforms as follows.

$$
\begin{gathered}
\Re_{1,1}(f)(x, y)=p \cdot v \cdot \int_{\mathbb{R}} \int_{\mathbb{R}} \frac{1}{u v} f(x-u, y-v) d u d v, \\
\mathfrak{R}_{1}(f)(x, y)=p \cdot v \cdot \int_{\mathbb{R}} \frac{1}{u} f(x-u, y) d u, \\
\mathfrak{R}_{2}(f)(x, y)=p \cdot v \cdot \int_{\mathbb{R}} \frac{1}{v} f(x, y-v) d v .
\end{gathered}
$$

Also, define $\mathfrak{R}_{0}$ as the identity map, namely

$$
\mathfrak{R}_{0}(f)(x, y)=f(x, y) .
$$

It is known (see $[14,18])$ that for all $f \in H^{p}(\mathbb{R} \times \mathbb{R}) \cap L^{2}(\mathbb{R} \times \mathbb{R})$,

$$
\|f\|_{H^{p}(\mathbb{R} \times \mathbb{R})} \simeq\left\|\Re_{1,1}(f)\right\|_{L^{p}(\mathbb{R} \times \mathbb{R})}+\sum_{m=0}^{2}\left\|\Re_{m}(f)\right\|_{L^{p}(\mathbb{R} \times \mathbb{R})} .
$$

\section{Proof of Theorems}

3.1. Proof of Theorem 1. By the Minkowski integral inequality and a scaling argument, we have

$$
\begin{aligned}
\left\|H_{\Phi}(f)\right\|_{H^{1}(\mathbb{R} \times \mathbb{R})} & \leq \int_{0}^{\infty} \int_{0}^{\infty} \frac{\Phi(s, t)}{s t}\left\|f\left(\frac{\dot{-},}{\bar{s}}, \frac{\dot{t}}{t}\right)\right\|_{H^{1}(\mathbb{R} \times \mathbb{R})} d s d t \\
& =\|f\|_{H^{1}(\mathbb{R} \times \mathbb{R})} \int_{0}^{\infty} \int_{0}^{\infty} \Phi(s, t) d s d t .
\end{aligned}
$$

This shows the sufficiency part.

Let $a$ be a function with support on $[-1,1]^{2}$, and be odd for both two variables and satisfies

(i) $a(x, y)=1 / 2$ for $(x, y) \in[1 / 4,1 / 2]^{2}$,

(ii) $1 / 2 \geq a\left(x_{1}, x_{2}\right) \geq 0$ on $[0,1]^{2}$. 
We can view such $a$ as a rectangular $(1,2,0)$-atom, so that by an easy computation, we know

$$
\|a\|_{H^{1}(\mathbb{R} \times \mathbb{R})} \preceq 1 .
$$

Suppose

$$
\int_{0}^{\infty} \int_{0}^{\infty} \Phi(s, t) d s d t=\infty,
$$

but $H_{\Phi}$ were bounded on $H^{1}$, we have

$$
\left\|H_{\Phi}(a)\right\|_{H^{1}} \preceq\|a\|_{H^{1}} \preceq 1 .
$$

On the other hand, by the Hilbert transform characterization of $H^{p}$, we have

$$
\begin{aligned}
\left\|H_{\Phi}(a)\right\|_{H^{1}} & \succeq\left\|H_{\Phi}(a)\right\|_{L^{1}} \\
& \geq \int_{0}^{1} \int_{0}^{1}\left|\int_{0}^{\infty} \int_{0}^{\infty} \frac{\Phi(s, t)}{s t} a\left(\frac{x}{s}, \frac{y}{t}\right) d s d t\right| d x_{1} d x_{2} \\
& =\int_{0}^{\infty} \int_{0}^{\infty} \frac{\Phi(s, t)}{s t}\left(\int_{0}^{1} \int_{0}^{1} a\left(\frac{x}{s}, \frac{y}{t}\right) d x d y\right) d s d t \\
& \geq \int_{0}^{\infty} \int_{0}^{\infty} \Phi(s, t) d s d t=\infty .
\end{aligned}
$$

This leads to a contradiction.

3.2. Proof of Theorem 2. Let $f(x, y) \in H^{1}(\mathbb{R} \times \mathbb{R})$ be even on both $x$ and $y$. Since $H^{1}$ is a subspace of $L^{1}$, we have

$$
f(x, y)=\sum \lambda_{j} A_{j}(x, y)
$$

for almost all $(x, y) \in \mathbb{R} \times \mathbb{R}$, where each $A_{j}$ is a $(1,0)$-atom and

$$
\sum\left|\lambda_{j}\right| \simeq\|f\|_{H^{1}(\mathbb{R} \times \mathbb{R})} .
$$

For a function $g$, we use the following notation.

$$
\begin{aligned}
g^{+,+} & =g, \quad g^{+,-}(x, y)=g(x,-y), \\
g^{-,+}(x, y) & =g(-x, y), \quad g^{-,-}(x, y)=g(-x,-y) .
\end{aligned}
$$

Similarly, for an open set $\Lambda$ in $\mathbb{R} \times \mathbb{R}$, we define

$$
\begin{aligned}
& \Lambda^{+,+}=\Lambda, \quad \Lambda^{+,-}=\{(x,-y):(x, y) \in \Lambda\}, \\
& \Lambda^{-,+}=\{(-x, y):(x, y) \in \Lambda\}, \quad \Lambda^{-,-}=\{(-x,-y):(x, y) \in \Lambda\} .
\end{aligned}
$$


Since $f$ is even on both $x$ and $y$ variables, we can write

$$
f=\frac{1}{4}\left(f^{+,+}+f^{+,-}+f^{-,+}+f^{-,-}\right) .
$$

Hence,

$$
f(x, y)=\sum \lambda_{j} B_{j}(x, y),
$$

where

$$
B_{j}=\frac{1}{4}\left(A_{j}^{+,+}+A_{j}^{+,-}+A_{j}^{-,+}+A_{j}^{-,-}\right) .
$$

Furthermore, we have

$$
\mathcal{H}(f)=\sum \lambda_{j} \mathcal{H}\left(B_{j}\right)
$$

Now, by the atomic characterization of $H^{1}(\mathbb{R} \times \mathbb{R})$, it suffices to show that each $\mathcal{H}\left(B_{j}\right)$ is again a (1,0)-atom.

For simplicity in the notation, we write $A=A_{j}$ and $B=B_{j}$. Let $\Omega$ be in the definition of $(1,0)$-atom for which $\operatorname{supp}(A) \subset \Omega$ and

$$
\|A\|_{L^{2}(\mathbb{R} \times \mathbb{R})} \leq|\Omega|^{-1 / 2} .
$$

The set

$$
\Omega^{\sharp}=\Omega^{+,+} \cup \Omega^{+,-} \cup \Omega^{-,+} \cup \Omega^{-,-}
$$

is an open set in $\mathbb{R} \times \mathbb{R}$ so that it is a union of disjoint open rectangles

$$
\Omega^{\sharp}=\cup_{\alpha} R_{\alpha} .
$$

We denote

$$
\Omega^{*}=\cup_{\alpha} 3 R_{\alpha} .
$$

Then $\Omega^{*}$ is also an open set in $\mathbb{R} \times \mathbb{R},\left|\Omega^{*}\right| \simeq|\Omega|$, and $\operatorname{supp}(B) \subset \Omega^{*}$.

Using the Minkowski integral inequality, we have

$$
\begin{aligned}
& \|\mathcal{H}(B)\|_{L^{2}(\mathbb{R} \times \mathbb{R})} \\
= & \left\|\int_{1}^{\infty} \int_{1}^{\infty} \frac{B(\dot{\bar{t}}, \dot{\bar{s}})}{t^{2} s^{2}} d t d s\right\|_{L^{2}(\mathbb{R} \times \mathbb{R})} \preceq\|B\|_{L^{2}(\mathbb{R} \times \mathbb{R})} \\
\leq & \frac{1}{4}\left\{\left\|A^{+,+}\right\|_{L^{2}(\mathbb{R} \times \mathbb{R})}+\left\|A^{+,-}\right\|_{L^{2}(\mathbb{R} \times \mathbb{R})}+\left\|A^{-,+}\right\|_{L^{2}(\mathbb{R} \times \mathbb{R})}+\left\|A^{-,-}\right\|_{L^{2}(\mathbb{R} \times \mathbb{R})}\right\}
\end{aligned}
$$


$\leq\left|\Omega^{*}\right|^{-1 / 2}$.

We know that

$$
A^{+,+}(x, y)=\sum_{R \in m(\Omega)} a_{R}^{+,+}(x, y),
$$

where each $a_{R}$ satisfies $\operatorname{supp}\left(a_{R}\right) \subset R$, and $R=I \times J$. We denote

$$
\begin{aligned}
& I^{+}=I, I^{-}=\{-x, \quad x \in I\}, \\
& J^{+}=J, J^{-}=\{-y, \quad y \in J\} .
\end{aligned}
$$

Then

$$
\begin{aligned}
& R^{+,+}=R, \quad R^{+,-}=I^{+} \times J^{-}, \\
& R^{-,+}=I^{-} \times J^{+}, \quad R^{-,-}=I^{-} \times J^{-} .
\end{aligned}
$$

With these definitions, we can write

$$
\begin{aligned}
& 4 \mathcal{H}(B)(x, y) \\
= & \sum_{R \in m(\Omega)}\left\{\mathcal{H}\left(a_{R}^{+,+}\right)(x, y)+\mathcal{H}\left(a_{R}^{+,-}\right)(x, y)+\mathcal{H}\left(a_{R}^{-,+}\right)(x, y)+\mathcal{H}\left(a_{R}^{-,-}\right)(x, y)\right\},
\end{aligned}
$$

where $a_{R}^{+,+}, a_{R}^{+,-}, a_{R}^{-,+}, a_{R}^{-,-}$are rectangular $(1,2,0)$-atoms and they satisfy

$$
\begin{aligned}
& \operatorname{supp}\left(a_{R}^{+,+}\right) \subset R^{+,+} \in m\left(\Omega^{+,+}\right), \quad \operatorname{supp}\left(a_{R}^{+,-}\right) \subset R^{+,-} \in m\left(\Omega^{+,-}\right), \\
& \operatorname{supp}\left(a_{R}^{-,+}\right) \subset R^{-,+} \in m\left(\Omega^{-,+}\right), \quad \operatorname{supp}\left(a_{R}^{-,-}\right) \subset R^{-,-} \in m\left(\Omega^{-,-}\right) .
\end{aligned}
$$

Now, write $R=R^{+,+}=I \times J=(\alpha, \beta) \times(c, d)$. We consider the following four cases:

Case 1: $0 \notin I, 0 \notin J$;

Case 2: $0 \notin I, 0 \in J$;

Case 3: $0 \in I, 0 \notin J$;

Case 4: $0 \in I, 0 \in J$.

In Case 1, without loss of generality, we assume $I=[\alpha, \beta] \subset(0, \infty)$ and $J=[c, d] \subset$ $(-\infty, 0)$. For any $x \notin[\alpha, \beta]$, if $x<\alpha$, clearly $\mathcal{H}\left(a_{R}\right)(x, y)=0$. If $x>\beta$, by the cancellation condition of $a_{R}$ we also see

$$
\mathcal{H}\left(a_{R}\right)(x, y)=\frac{1}{x y} \int_{0}^{x} \int_{0}^{y} a_{R}(t, s) d t d s=\frac{1}{x y} \int_{\alpha}^{\beta} \int_{0}^{y} a_{R}(t, s) d t d s=0 .
$$

This indicates that for any $(x, y) \notin R=I \times J, \mathcal{H}\left(a_{R}\right)(x, y)=0$. Similarly, we can show

$$
\mathcal{H}\left(a_{R}^{+,-}\right)(x, y)=0, \quad \text { if }(x, y) \notin R^{+,-},
$$




$$
\begin{aligned}
& \mathcal{H}\left(a_{R}^{-,+}\right)(x, y)=0, \quad \text { if }(x, y) \notin R^{-,+}, \\
& \mathcal{H}\left(a_{R}^{-,-}\right)(x, y)=0, \quad \text { if }(x, y) \notin R^{-,-} .
\end{aligned}
$$

Also, using the support condition and the Fubini theorem, it is easy to check that

$$
\begin{aligned}
& \int_{\mathbb{R}} \mathcal{H}\left(a_{R}^{+,+}\right)(x, y) d x=\int_{\alpha}^{\beta} \int_{1}^{\infty} \int_{1}^{\infty} \frac{1}{s^{2} t^{2}} a\left(\frac{x}{t}, \frac{y}{s}\right) d t d s d x=0, \\
& \int_{\mathbb{R}} \mathcal{H}\left(a_{R}^{+,+}\right)(x, y) d y=\int_{c}^{d} \int_{1}^{\infty} \int_{1}^{\infty} \frac{1}{s^{2} t^{2}} a\left(\frac{x}{t}, \frac{y}{s}\right) d t d s d y=0 .
\end{aligned}
$$

This shows that $\mathcal{H}\left(a_{R}^{+,+}\right)$satisfies (2-i) and (2-ii).

Similarly, we can show that $\mathcal{H}\left(a_{R}^{+,-}\right), \mathcal{H}\left(a_{R}^{-,+}\right), \mathcal{H}\left(a_{R}^{-,-}\right)$satisfy (2-i) and (2-ii). Since it is shown

$$
\begin{aligned}
& \operatorname{supp}\left(\mathcal{H}\left(a_{R}^{+,+}\right)\right) \subset 3 R^{+,+} \\
& \operatorname{supp}\left(\mathcal{H}\left(a_{R}^{+,-}\right)\right) \subset 3 R^{+,-} \\
& \operatorname{supp}\left(\mathcal{H}\left(a_{R}^{-,+}\right)\right) \subset 3 R^{-,+} \\
& \operatorname{supp}\left(\mathcal{H}\left(a_{R}^{-,-}\right)\right) \subset 3 R^{-,-},
\end{aligned}
$$

we conclude that in Case 1 , all $\mathcal{H}\left(a_{R}^{+,+}\right), \mathcal{H}\left(a_{R}^{+,-}\right), \mathcal{H}\left(a_{R}^{-,+}\right), \mathcal{H}\left(a_{R}^{-,-}\right)$are rectangular $(1,2,0)$-atoms.

In Case 2, without loss of generality, we assume $I=[\alpha, \beta] \subset(0, \infty)$ and $J=[-c, c]$. We now write

$$
\begin{aligned}
& \left\{\mathcal{H}\left(a_{R}^{+,+}\right)(x, y)+\mathcal{H}\left(a_{R}^{+,-}\right)(x, y)+\mathcal{H}\left(a_{R}^{-,+}\right)(x, y)+\mathcal{H}\left(a_{R}^{-,-}\right)(x, y)\right\} \\
= & \mathcal{H}\left(\left(a_{R}^{+,+}+a_{R}^{+,-}\right) \chi_{\mathbb{R} \times[-c, 0)}\right)(x, y)+\mathcal{H}\left(\left(a_{R}^{+,+}+a_{R}^{+,-}\right) \chi_{\mathbb{R} \times(0, c]}\right)(x, y) \\
& +\mathcal{H}\left(\left(a_{R}^{-,+}+a_{R}^{-,-}\right) \chi_{\mathbb{R} \times[-c, 0]}\right)(x, y)+\mathcal{H}\left(\left(a_{R}^{-,+}+a_{R}^{-,-}\right) \chi_{\mathbb{R} \times[0, c]}\right)(x, y) .
\end{aligned}
$$

Since both $a_{R}^{+,+}+a_{R}^{+,-}$and $a_{R}^{-,+}+a_{R}^{-,-}$are even on $y$, it is easy to check

$$
\begin{aligned}
& \int_{\mathbb{R}}\left(a_{R}^{+,+}+a_{R}^{+,-}\right)(x, y) \chi_{\mathbb{R} \times[-c, 0]}(x, y) d x=0=\int_{\mathbb{R}}\left(a_{R}^{+,+}+a_{R}^{+,-}\right)(x, y) \chi_{\mathbb{R} \times[0, c]}(x, y) d y, \\
& \int_{\mathbb{R}}\left(a_{R}^{-,+}+a_{R}^{-,-}\right)(x, y) \chi_{\mathbb{R} \times[-c, 0]}(x, y) d x=0=\int_{\mathbb{R}}\left(a_{R}^{-,+}+a_{R}^{-,-}\right)(x, y) \chi_{\mathbb{R} \times[0, c]}(x, y) d y .
\end{aligned}
$$


The known result for Case 1 implies that $\mathcal{H}\left(\left(a_{R}^{+,+}+a_{R}^{+,-}\right) \chi_{\mathbb{R} \times[-c, 0]}\right)$, $\mathcal{H}\left(\left(a_{R}^{+,+}+a_{R}^{+,-}\right) \chi_{\mathbb{R} \times[0, c]}\right), \mathcal{H}\left(\left(a_{R}^{-,+}+a_{R}^{-,-}\right) \chi_{\mathbb{R} \times[-c, 0]}\right), \mathcal{H}\left(\left(a_{R}^{-,+}+a_{R}^{-,-}\right) \chi_{\mathbb{R} \times[0, c]}\right)$ satisfy (2-i) and (2-ii). Thus, they are all rectangular (1,2,0)-atoms .

In Case 3, without loss of generality, we assume $I=(-\alpha, \alpha)$ and $J=(c, d)$ $\subset(0, \infty)$. Then both $a_{R}^{+,+}+a_{R}^{-,+}$and $a_{R}^{+,-}+a_{R}^{-,-}$are even in $x$. Similar to the discussion in Case 3, we know that $\mathcal{H}\left(\left(a_{R}^{+,+}+a_{R}^{-,+}\right) \chi_{(-\alpha, 0) \times \mathbb{R}}\right), \mathcal{H}\left(\left(a_{R}^{+,+}+a_{R}^{-,+}\right) \chi_{(0, \alpha) \times \mathbb{R}}\right)$, $\mathcal{H}\left(\left(a_{R}^{+,-}+a_{R}^{-,-}\right) \chi_{(-\alpha, 0) \times \mathbb{R}}\right), \quad \mathcal{H}\left(\left(a_{R}^{+,-}+a_{R}^{-,-}\right) \chi_{(0, \alpha) \times \mathbb{R}}\right)$ are rectangular $(1,2,0)$ atoms.

In Case 4, without loss of generality, we assume $I=(-\alpha, \alpha)$ and $J=[-c, c]$. Then, we know that the function

$$
\mathrm{a}_{R}=a_{R}^{+,+}+a_{R}^{+,-}+a_{R}^{-,+}+a_{R}^{-,-}
$$

is even on both $x$ and $y$ variables. With the same reason as that of Case 2, it is easy to see that all $\mathcal{H}\left(\mathrm{a}_{R} \chi_{[-\alpha, 0] \times[-c, 0]}\right), \mathcal{H}\left(\mathrm{a}_{R} \chi_{[-\alpha, 0] \times[0, c]}\right), \mathcal{H}\left(\mathrm{a}_{R} \chi_{[0, \alpha] \times[0, c]}\right), \mathcal{H}\left(\mathrm{a}_{R} \chi_{[0, \alpha] \times[-c, 0]}\right)$ are rectangular $(1,2,0)$-atoms.

We now write

$$
\begin{aligned}
& a_{R}^{+,+}=a_{R^{+,+}}, \quad a_{R}^{+,-}=a_{R^{+,-},}, \\
& a_{R}^{-,+}=a_{R^{-,+}}, \quad a_{R}^{-,-}=a_{R^{-,-}},
\end{aligned}
$$

and denote

$$
\begin{aligned}
& \widetilde{\mathfrak{R}_{1}}=\left\{R^{+,+}, R^{+,-}, R^{-,+}, R^{-,-}: \text {if } R^{+,+} \text {is in Case } 1\right\}, \\
& \widetilde{\mathfrak{R}_{2}}=\left\{R^{+,+}, R^{+,-}, R^{-,+}, R^{-,-}: \text {if } R^{+,+} \text {is in Case } 2\right\}, \\
& \widetilde{\mathfrak{R}_{3}}=\left\{R^{+,+}, R^{+,-}, R^{-,+}, R^{-,-}: \text {if } R^{+,+} \text {is in Case } 3\right\}, \\
& \widetilde{\mathfrak{R}_{4}}=\left\{R^{+,+}, R^{+,-}, R^{-,+}, R^{-,-}: \text {if } R^{+,+} \text {is in Case } 4\right\},
\end{aligned}
$$

and use the notation $\widetilde{\Re_{j}} \in m\left(\Omega^{*}\right), j=1,2,3,4$ to mean that $R^{+,+}$is in Case $j$, and

$$
R^{+,+}, R^{+,-}, R^{-,+}, R^{-,-} \in m\left(\Omega^{*}\right) .
$$

By this notation and the above discussion, we have $\operatorname{supp}(\mathcal{H}(B)) \subset \Omega^{*}$, and

$$
\begin{aligned}
& 4 \mathcal{H}(B)(x, y) \\
= & \sum_{\widetilde{\mathfrak{R}_{1}} \in m\left(\Omega^{*}\right)}\left\{\mathcal{H}\left(a_{R^{+,+}}\right)+\mathcal{H}\left(a_{R^{+,-}}\right)+\mathcal{H}\left(a_{R^{-,+}}\right)+\mathcal{H}\left(a_{R^{-,-}}\right)\right\} \\
& +\sum_{\widetilde{\Re_{2}} \in m\left(\Omega^{*}\right)}\left\{\mathcal{H}\left(\left(a_{R^{+,+}}+a_{R^{+,-}}\right) \chi_{\mathbb{R} \times[-c, 0)}\right)+\mathcal{H}\left(\left(a_{R^{+,+}}+a_{R^{+,-}}\right) \chi_{\mathbb{R} \times(0, c]}\right)\right.
\end{aligned}
$$




$$
\begin{aligned}
& \left.+\mathcal{H}\left(\left(a_{R^{-,+}}+a_{R^{-,-}}\right) \chi_{\mathbb{R} \times[-c, 0)}\right)+\mathcal{H}\left(\left(a_{R^{-,+}}+a_{R^{-,-}}\right) \chi_{\mathbb{R} \times(0, c]}\right)\right\} \\
& +\sum_{\widetilde{\mathfrak{R}_{3}} \in m\left(\Omega^{*}\right)}\left\{\mathcal{H}\left(\left(a_{R^{-,+}}+a_{R^{+,+}}\right) \chi_{[-\alpha, 0) \times \mathbb{R}}\right)+\mathcal{H}\left(\left(a_{R^{-,+}}+a_{R^{+,+}}\right) \chi_{(0, \alpha) \times \mathbb{R}}\right)\right. \\
& \left.+\mathcal{H}\left(\left(a_{R^{-,-}}+a_{R^{+,-}}\right) \chi_{[-\alpha, 0) \times \mathbb{R}}\right)+\mathcal{H}\left(\left(a_{R^{-,-}}+a_{R^{+,-}}\right) \chi_{(0, a) \times \mathbb{R}}\right)\right\} \\
& +\underset{\widetilde{\mathfrak{R}_{4}} \in m\left(\Omega^{*}\right)}{ }\left\{\mathcal{H}\left(\mathrm{a}_{R} \chi_{[-a, 0) \times[-c, 0)}\right)+\mathcal{H}\left(\mathrm{a}_{R} \chi_{[-\alpha, 0) \times(0, c]}\right)\right. \\
& \left.+\mathcal{H}\left(\mathrm{a}_{R} \chi_{(0, \alpha] \times(0, c]}\right)+\mathcal{H}\left(\mathrm{a}_{R} \chi_{(0, \alpha] \times[-c, 0)}\right)\right\} .
\end{aligned}
$$

Finally, since $\mathcal{H}$ is bounded on $L^{2}(\mathbb{R} \times \mathbb{R})([17])$, we easily obtain

$$
\begin{aligned}
& \sum_{\widetilde{\mathfrak{R}_{1}} \in m\left(\Omega^{*}\right)}\left\{\left\|\mathcal{H}\left(a_{R^{+,+}}\right)\right\|_{L^{2}}^{2}+\left\|\mathcal{H}\left(a_{R^{+,-}}\right)\right\|_{L^{2}}^{2}+\left\|\mathcal{H}\left(a_{R^{-,+}}\right)\right\|_{L^{2}}^{2}+\left\|\mathcal{H}\left(a_{R^{-,-}}\right)\right\|_{L^{2}}^{2}\right\} \\
& +\sum_{\widetilde{\Re_{2}} \in m\left(\Omega^{*}\right)}\left\{\left\|\mathcal{H}\left(\left(a_{R^{+,+}}+a_{R^{+,-}}\right) \chi_{\mathbb{R} \times[-c, 0)}\right)\right\|_{L^{2}}^{2}+\left\|\mathcal{H}\left(\left(a_{R^{+,+}}+a_{R^{+,-}}\right) \chi_{\mathbb{R} \times(0, c]}\right)\right\|_{L^{2}}^{2}\right. \\
& \left.+\left\|\mathcal{H}\left(\left(a_{R^{-,}+}+a_{R^{-,-}}\right) \chi_{\mathbb{R} \times[-c, 0)}\right)\right\|_{L^{2}}^{2}+\left\|\mathcal{H}\left(\left(a_{R^{-,+}}+a_{R^{-,-}}\right) \chi_{\mathbb{R} \times(0, c]}\right)\right\|_{L^{2}}^{2}\right\} \\
& +\sum_{\widetilde{\Re_{3}} \in m\left(\Omega^{*}\right)}\left\{\left\|\mathcal{H}\left(\left(a_{R^{-,+}}+a_{R^{+,+}}\right) \chi_{[-\alpha, 0) \times \mathbb{R}}\right)\right\|_{L^{2}}^{2}+\left\|\mathcal{H}\left(\left(a_{R^{-,+}}+a_{R^{+,+}}\right) \chi_{(0, \alpha) \times \mathbb{R}}\right)\right\|_{L^{2}}^{2}\right. \\
& \left.+\left\|\mathcal{H}\left(\left(a_{R^{-,-}}+a_{R^{+,-}}\right) \chi_{[-\alpha, 0) \times \mathbb{R}}\right)\right\|_{L^{2}}^{2}+\left\|\mathcal{H}\left(\left(a_{R^{-,-}}+a_{R^{+,-}}\right) \chi_{(0, a) \times \mathbb{R}}\right)\right\|_{L^{2}}^{2}\right\} \\
& +\sum_{\widetilde{\mathfrak{R}_{4}} \in m\left(\Omega^{*}\right)}\left\{\left\|\mathcal{H}\left(\mathrm{a}_{R} \chi_{[-a, 0) \times[-c, 0)}\right)\right\|_{L^{2}}^{2}+\left\|\mathcal{H}\left(\mathrm{a}_{R} \chi_{[-\alpha, 0) \times(0, c]}\right)\right\|_{L^{2}}^{2}\right. \\
& \left.+\left\|\mathcal{H}\left(\mathrm{a}_{R} \chi_{(0, \alpha] \times(0, c]}\right)\right\|_{L^{2}}^{2}+\left\|\mathcal{H}\left(\mathrm{a}_{R} \chi_{(0, \alpha] \times[-c, 0)}\right)\right\|_{L^{2}}^{2}\right\} \preceq\left|\Omega^{*}\right|^{-1} .
\end{aligned}
$$

Combining all estimates, we know that $\mathcal{H}(B)$ is a $(1,0)$-atom. This completes the proof.

\subsection{Proof of Theorem 3. First, we note}

$$
\begin{aligned}
\mathcal{H}_{(k)} f(x, y) & =\frac{1}{x^{k+1} y^{k+1}} \int_{0}^{x} \int_{0}^{y} t^{k} s^{k} f(t, s) d t d s \\
& =\int_{0}^{1} \int_{0}^{1} t^{k} s^{k} f(x t, y s) d t d s=\int_{1}^{\infty} \int_{1}^{\infty} \frac{1}{t^{k+2} s^{k+2}} f(x / t, y / s) d t d s \\
& =\int_{1}^{\infty} \frac{1}{s^{k+2}}\left(\int_{0}^{1} t^{k} f(x t, y / s) d t\right) d s .
\end{aligned}
$$


By the Minkowski integral inequality and a scaling argument, for any $f \in L^{2}$, we have

$$
\begin{aligned}
\left\|\mathcal{H}_{(k)}(f)\right\|_{L^{2}(\mathbb{R} \times \mathbb{R})} & \leq \int_{0}^{\infty} \int_{0}^{\infty} \frac{\Phi(s, t)}{s t}\left\|f\left(\frac{\dot{-},}{s}, \frac{\cdot}{t}\right)\right\|_{L^{2}(\mathbb{R} \times \mathbb{R})} d s d t \\
& =\|f\|_{L^{2}(\mathbb{R} \times \mathbb{R})} \int_{0}^{\infty} \int_{0}^{\infty} \frac{\Phi(s, t)}{s t} s^{1 / 2} t^{1 / 2} d s d t,
\end{aligned}
$$

where

$$
\Phi(s, t)=\chi_{(1, \infty) \times(1, \infty)}(s, t) s^{-1-k} t^{-1-k} .
$$

Hence, the operator $\mathcal{H}_{(k)}$ is bounded on $L^{2}$. We first show that $\mathcal{H}_{(k)}$ is bounded from $H^{p}(\mathbb{R} \times \mathbb{R})$ to $L^{p}(\mathbb{R} \times \mathbb{R})$. To this end, we only need to check that $\mathcal{H}_{(k)}$ satisfies the condition in Theorem A.

Let $a$ be a rectangular $(p, 2, k)$-atom with support in $R=I \times J$, where $I=[\alpha, \beta]$ and $J=[c, d]$. To verify that $\mathcal{H}_{(k)}(a)$ satisfies the condition in Theorem A, it suffices to consider the following three cases:

Case 1: $0 \notin I, 0 \notin J$;

Case 2: $0 \notin I, 0 \in J$;

Case 3: $0 \in I, 0 \in J$.

In Case 1, without loss of generality, we assume $I=[\alpha, \beta] \subset(0, \infty)$ and $J=[c, d] \subset$ $(-\infty, 0)$. For any $(x, y) \notin I \times J$, as argument in the proof of Theorem 2 , it is easy to see that

$$
\mathcal{H}_{(k)}(a)(x, y)=\frac{1}{x^{k+1} y^{k+1}} \int_{0}^{x} \int_{0}^{y} t^{k} s^{k} a(t, s) d t d s=0 .
$$

This indicates that $H_{(k)}(a)$ is also supported in $R=I \times J$. Hence

$$
\int_{(\gamma R)^{C}}\left|\mathcal{H}_{(k)}(a)(x, y)\right|^{p} d x d y=0 .
$$

In Case 2, without loss of generality, we assume $I=[\alpha, \beta] \subset(0, \infty)$ and $J=[-r, r]$. We write

$$
\begin{aligned}
\mathcal{H}_{(k)}(a)(x, y) & =\frac{1}{x^{k+1}} \int_{0}^{x} t^{k} \int_{1}^{\infty} s^{-k-2} a(t, y / s) d t d s \\
& =\sum_{j=0}^{\infty} 2^{-j \sigma} \frac{1}{x^{k+1}} \int_{0}^{x} t^{k} \int_{2^{j}}^{2^{j+1}} 2^{j \sigma} s^{-k-2} a(t, y / s) d t d s \\
& =\sum_{j=0}^{\infty} 2^{-j \sigma} A_{j}(x, y)
\end{aligned}
$$

where

$$
\sigma=k-1 / p+1
$$


is a positive number. The support of $A_{j}$ is in

$$
R_{j}=I \times\left[-2^{j+1} r, 2^{j+1} r\right] .
$$

To check the size condition of $A_{j}$, we write

$$
A_{j}(x, y)=\int_{1}^{\infty} t^{-k-2} \int_{2^{j}}^{2^{j+1}} 2^{j \sigma} s^{-k-2} a(x / t, y / s) d t d s .
$$

By the Minkowski integral inequality and a scaling argument, we have

$$
\begin{aligned}
\left\|A_{j}\right\|_{L^{2}} & \preceq \int_{1}^{\infty} t^{-k-2} \int_{2^{j}}^{2^{j+1}} 2^{j \sigma} s^{-k-2}\|a(\cdot / t, \cdot / s)\|_{L^{2}} d t d s \\
& \preceq\|a\|_{L^{2}} \int_{2^{j}}^{2^{j+1}} s^{-k-2+1 / 2+\sigma} d s \preceq 2^{-k j+\sigma j-j / 2}|R|^{1 / 2-1 / p} .
\end{aligned}
$$

Thus indicates that,

$$
\left\|A_{j}\right\|_{L^{2}} \preceq\left|R_{j}\right|^{1 / 2-1 / p}
$$

Also, it is easy to check

$$
\int_{\mathbb{R}} x^{\alpha} A_{j}(x, y) d x=0 \text { for all } y \in \mathbb{R}
$$

and

$$
\int_{\mathbb{R}} A_{j}(x, y) y^{\alpha} d y=0 \text { for all } x \in \mathbb{R}
$$

for all $\alpha=0,1,2, \ldots, s$. Thus, $A_{j}(x, y)$ is a rectangular $(p, 2, k)$-atom with support in $R_{j}$.

For any $\gamma \geq 2$, we write

$$
\mathcal{H}_{(k)}(a)(x, y)=\sum_{2^{j+1}>\gamma} 2^{-j \sigma} A_{j}(x, y)+\sum_{2^{j+1} \leq \gamma} 2^{-j \sigma} A_{j}(x, y) .
$$

Note

$$
\operatorname{supp}\left(A_{j}\right) \cap(\gamma R)^{C}=\phi
$$

if $2^{j+1} \leq \gamma$. We have

$$
\begin{aligned}
& \int_{(\gamma R)^{C}}\left|\mathcal{H}_{(k)}(a)(x, y)\right|^{p} d x d y \\
\preceq & \sum_{2^{j+1}>\gamma} 2^{-j \sigma p} \int_{(\gamma R)^{C}}\left|A_{j}(x, y)\right|^{p} d x d y
\end{aligned}
$$




$$
\preceq \sum_{2^{j+1}>\gamma} 2^{-j \sigma p} \preceq \gamma^{-\sigma p} .
$$

In Case 3, without loss of generality, we assume $I=[-\rho, \rho]$ and $J=[-r, r]$. We write

$$
\begin{aligned}
\mathcal{H}_{(k)}(a)(x, y) & =\int_{1}^{\infty} s^{-k-2} \int_{1}^{\infty} t^{-k-2} a(x / s, y / t) d t d s \\
& =\sum_{i=0}^{\infty} \sum_{j=0}^{\infty} 2^{-i \sigma} 2^{-j \sigma} \int_{2^{i}}^{2^{i+1}} 2^{j \sigma} s^{-k-2} \int_{2^{j}}^{2^{j+1}} 2^{i \sigma} t^{-k-2} a(x / s, y / t) d s d t \\
& =\sum_{i=0}^{\infty} \sum_{j=0}^{\infty} 2^{-i \sigma} 2^{-j \sigma} A_{i, j}(x, y) .
\end{aligned}
$$

It is easy to check that the support of $A_{i, j}$ is in

$$
R_{i, j}=\left[-2^{i+1} \rho, 2^{i+1} \rho\right] \times\left[-2^{j+1} r, 2^{j+1} r\right] .
$$

Again, by the Minkowski integral inequality and a scaling argument, we have

$$
\begin{aligned}
\left\|A_{i, j}\right\|_{L^{2}} & \preceq \int_{2^{i}}^{2^{i+1}} s^{-k-2+\sigma} \int_{2^{j}}^{2^{j+1}} t^{-k-2+\sigma}\|a(\cdot / t, \cdot / s)\|_{L^{2}} d t d s \\
& \preceq\|a\|_{L^{2}} \int_{2^{i}}^{2^{i+1}} s^{-k-2+1 / 2+\sigma} d s \int_{2^{j}}^{2^{j+1}} t^{-k-2+1 / 2+\sigma} d t \preceq\left|R_{i, j}\right|^{1 / 2-1 / p} .
\end{aligned}
$$

Thus, it is easy to check that each $A_{i, j}$ is a rectangular $(p, 2, k)$-atom. We now write

$$
\begin{aligned}
\mathcal{H}_{(k)}(a)(x, y) & =\sum_{2^{i+1}>\gamma} \sum_{2^{j+1} \leq \gamma} 2^{-i \sigma} 2^{-j \sigma} A_{i, j}(x, y)+\sum_{2^{i+1} \leq \gamma} \sum_{2^{j+1} \leq \gamma} 2^{-i \sigma} 2^{-j \sigma} A_{i, j}(x, y) \\
& +\sum_{2^{i+1} \leq \gamma} \sum_{2^{j+1}>\gamma} 2^{-i \sigma} 2^{-j \sigma} A_{i, j}(x, y)+\sum_{2^{i+1}>\gamma} \sum_{2^{j+1}>\gamma} 2^{-i \sigma} 2^{-j \sigma} A_{i, j}(x, y) .
\end{aligned}
$$

Using the same argument as that in Case 2, we have

$$
\int_{(\gamma R)^{C}}\left|\mathcal{H}_{(k)}(a)(x, y)\right|^{p} d x d y \preceq \gamma^{-\sigma p} .
$$

Thus, by Theorem A, we know that $\mathcal{H}_{(k)}$ is bounded from $H^{p}(\mathbb{R} \times \mathbb{R})$ to $L^{p}(\mathbb{R} \times \mathbb{R})$.

Finally, by the Hilbert transform characterization of $H^{p}$, we know that for $f \in L^{2} \cap H^{p}$,

$$
\left\|\mathcal{H}_{(k)}(f)\right\|_{H^{p}(\mathbb{R} \times \mathbb{R})} \simeq\left\|\Re_{1,1} \mathcal{H}_{(k)}(f)\right\|_{L^{p}(\mathbb{R} \times \mathbb{R})}+\sum_{m=0}^{2}\left\|\Re_{m} \mathcal{H}_{(k)}(f)\right\|_{L^{p}(\mathbb{R} \times \mathbb{R})} .
$$


Following the same argument as in [13], we have

$$
\mathfrak{R}_{1,1} \mathcal{H}_{(k)}(f)=\mathcal{H}_{(k)}\left(\Re_{1,1} f\right)
$$

and

$$
\mathfrak{R}_{m} \mathcal{H}_{(k)}(f)=\mathcal{H}_{(k)}\left(\Re_{m} f\right), \quad m=0,1,2
$$

(one also easily sees the above commutating relations for Hausdorff operators and Hilbert transforms, since the Hilbert transforms commute with the dilation).

Thus, for $k>1 / p-1$,

$$
\begin{aligned}
\left\|\mathcal{H}_{(k)}(f)\right\|_{H^{p}(\mathbb{R} \times \mathbb{R})} & \preceq\left\|\Re_{1,1}(f)\right\|_{H^{p}(\mathbb{R} \times \mathbb{R})}+\sum_{m=0}^{2}\left\|\Re_{m}(f)\right\|_{H^{p}(\mathbb{R} \times \mathbb{R})} \\
& \preceq\|f\|_{H^{p}(\mathbb{R} \times \mathbb{R})} .
\end{aligned}
$$

The last inequality is because that the Hilbert transforms are bounded on $H^{p}(\mathbb{R} \times \mathbb{R})$.

ACKNOWLEDGMEnT. The authors would like to thank the referee for careful checking the manuscript and correcting some mistakes in an earlier version of the paper.

\section{References}

[ 1 ] J. Chen, D. FAn and J. Li, Hausdorff Operators on Function Spaces, Chinese Ann. Math. Ser. B 33 (2012), 537-556.

[ 2 ] J. CHEN, D. FAN and C. ZHANG, Boundedness of Hausdorff operators on some product Hardy type spaces, Appl. Math. J. Chinese Univ. Ser. B 27 (2012), 114-126.

[ 3 ] R.R. Coifman and G. Weiss, Extensions of Hardy spaces and their use in analysis, Bull. Amer. Math. Soc. 83 (1977), 569-645.

[ 4 ] D. Deng and Y. Han, $H^{p}$ Space Theory (in Chinese), Beijing University Press, Beijing, 1992.

[ 5 ] D. FAN and X. LIN, Hausdorff operator on real Hardy spaces, preprint.

[6] R. FefFerman, Some recent developments in fourier analysis and $H^{p}$ theory on product domains-II, Function Spaces and Applications, Springer, Berlin Heidelberg, 1988, 44-51.

[ 7 ] R. FefFerman, Harmonic analysis on product spaces, Ann. of Math. 126 (1987), 109-130.

[ 8 ] G.H. Hardy, J.E. Littlewood and G. Pólya, Inequalities, Cambridge University Press, Cambridge, 1952.

[ 9] Y. KAnJin, The Hausdorff operators on the real Hardy spaces $H^{1}(\mathbb{R})$, Studia Math. 148 (2001), 37-45.

[10] E. LIFLYAND, Boundedness of multidimensional Hausdorff operators on $H^{1}\left(\mathbb{R}^{n}\right)$, Acta Sci. Math. (Szeged) 74 (2008), 845-851.

[11] E. LifLyAnd and A. MiYACHI, Boundedness of the Hausdorff operators in $H^{p}$ spaces, $0<p<1$, Studia Math. 194 (2009), 279-292.

[12] E. LIFLYAND and F. MóRICZ, The Hausdorff operator is bounded on the real Hardy space $H^{1}(\mathbb{R})$, Proc. Amer. Math. Soc. 128 (2000), 1391-1396.

[13] E. LIFLYAND and F. MóRICZ, Commuting relations for Hausdorff operators and Hilbert transforms on real Hardy spaces, Acta Math. Hungar. 97 (2002), 133-143. 
[14] E. LiflyAnd and F. Móricz, The multi-parameter Hausdorff operator is bounded on the product Hardy space $H^{11}(\mathbb{R} \times \mathbb{R})$, Analysis 21 (2001), 107-118.

[15] X. LIN and L. Sun, Some estimates on Hausdorff operator, Acta Sci. Math. (Szeged) 78 (2012), 669-681.

[16] F. MóRICZ, Multivariate Hausdorff operators on the spaces $H^{1}\left(\mathbb{R}^{n}\right)$ and $B M O\left(\mathbb{R}^{n}\right)$, Analysis Math. 31 (2005), 31-41.

[17] B.G. Pachpatte, On multivariate Hardy type inequalities, An. Stiint. Univ. Al. I. Cuza Iasi. Mat. (N.S.) 38 (1992), 355-361.

[18] F. WEISZ, Singular integrals on product domains, Arch. Math. 77 (2001), 328-336.

[19] X. WU and J. ChEn, Best constant for Hausdorff operators on $n$-dimensional product spaces, Sci. China Math. 57 (2014), 569-578.

[20] X. ZHU and J. CHEN, Integrability of general product Hardy operators on the product Hardy space, J. Chinese Univ. Ser. B 27 (2012), 225-233.

\author{
Present Addresses: \\ DASHAN FAN \\ DePARTMENT OF MATHEMATiCAL SCIENCES, \\ UNIVERSITY OF WISCONSIN-MILWAUKEE, \\ MiLWAUKEE, WI 53201, USA. \\ e-mail: fan@uwm.edu
}

FAYOU ZHAO

DEPARTMENT OF MATHEMATICS,

SHANGHAI UNIVERSITY,

Shanghai 200444, P.R. CHINA.

e-mail: fyzhao@shu.edu.cn 